\title{
Prophylactic effect of flavanol rich preparation metabolites in promoting resilience to a mouse model of social stress
}

\author{
Jun Wang $\mathbb{1}^{1,2}$, Danyue Zhao ${ }^{3}$, Simoni Tiano ${ }^{1}$, Adelaida Esteban-Fernández', Bo Yuan³ ${ }^{3}$ Chad Smith',

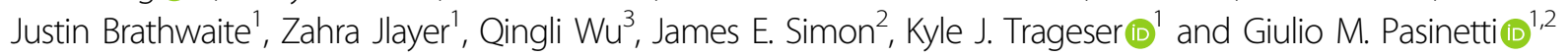

\begin{abstract}
Major depressive disorder (MDD) is a leading cause of disability, and there is an urgent need for new therapeutics. Stress-mediated induction of pro-inflammation in the periphery contributes to depression-like behaviors both in humans and in experimental models. Inflammatory cytokine interleukin-6 (IL-6) has emerged as a potential therapeutic target. Our studies demonstrated that metabolism of flavanol rich cocoa preparation (FRP) led to the accumulation of select phenolic acids that may contribute to its anti-inflammatory activity. Using a repeated social defeat stress (RSDS) model of depression, we showed that oral administration of FRP attenuates susceptibility to RSDS-mediated depression, supporting the further development of FRP as a novel therapeutic for the treatment of stress disorders and anxiety in humans.
\end{abstract}

\section{Introduction}

Major depressive disorder (MDD) takes an enormous toll on daily life and normal functioning. Currently, available treatments for depression target neurochemical or neurobiological mechanisms identified retrospectively following the discovery of the drug's initial antidepressant efficacy ${ }^{1}$. As conventional pharmacological treatments are estimated to produce temporary remission in $<50 \%$ of patients and are often accompanied by a wide range of adverse events ${ }^{2,3}$, there is a need for a wider spectrum of novel therapeutics to target newly discovered underlying disease mechanisms.

MDD is a multifaceted disease and the etiology is not completely understood. The contribution of the central nervous system (CNS) in the pathophysiology of MDD has been extensively studied ${ }^{4-7}$. In recent decades,

\footnotetext{
Correspondence: Giulio M. Pasinetti (giulio.pasinetti@mssm.edu)

'Department of Neurology, Icahn School of Medicine at Mount Sinai, New York, NY 10029, USA

${ }^{2}$ Geriatric Research, Education and Clinical Center, James J. Peters Veterans

Affairs Medical Center, Bronx, New York, NY 10468, USA
}

Full list of author information is available at the end of the article peripheral inflammation has been recognized as one of the emerging mechanisms of MDD and potential therapeutic targets ${ }^{8-13}$. Although many neuroimmune factors have been implicated in depressive disorders, studies in humans suggest that elevation of peripheral IL-6 is one factor that is most consistently observed ${ }^{14-16}$. Moreover, in a repeated social defeat stress (RSDS) mouse model of depression, it was reported that mice show individual differences in IL-6 production in response to RSDS, and that this intrinsic immune characteristic predicts susceptibility versus resilience to RSDS. Sequestering and neutralizing peripheral IL-6 using monoclonal antibodies significantly promotes resilience to RSDS-induced depression phenotypes ${ }^{17}$. While the mechanisms by which peripheral IL-6 may modulate depression phenotypes is currently under intense investigation, evidence suggest that in both human and in rodent models of depression, chronic social stress alters blood vessel ultrastructure, and in combination with stress-induced peripheral inflammation, increases blood brain barrier (BBB) leakiness that allows the infiltration of inflammatory molecules into the brain $^{18}$. Once IL- 6 crosses the

\section{(c) The Author(s) 2020}

(c) (i) Open Access This article is licensed under a Creative Commons Attribution 4.0 International License, which permits use, sharing, adaptation, distribution and reproduction cc) in any medium or format, as long as you give appropriate credit to the original author(s) and the source, provide a link to the Creative Commons license, and indicate if changes were made. The images or other third party material in this article are included in the article's Creative Commons license, unless indicated otherwise in a credit line to the material. If material is not included in the article's Creative Commons license and your intended use is not permitted by statutory regulation or exceeds the permitted use, you will need to obtain permission directly from the copyright holder. To view a copy of this license, visit http://creativecommons.org/licenses/by/4.0/. 
$\mathrm{BBB}$, it may alter neuroplasticity and modulate brain activity by acting directly on neurons, or indirectly through modulation of microglia and/or other CNS immune cells ${ }^{19}$. These studies suggest that modulation of IL-6 and associated immune signaling pathways may provide a novel therapeutic strategy to prevent and/or treat depression.

Cocoa is the dried seed of Theobroma cacao, and is a rich source of polyphenols, particularly epicatechins ${ }^{20,21}$. Previously, we found an epicatechin-rich grape polyphenol extract that was highly effective in protecting against the onset and/or progression of multiple, diverse neurological, psychological, and metabolic disorders in animal models, mainly due to its bioactivities in modulating synaptic function ${ }^{22-28}$. Published evidence suggests cocoa consumption (in the form of chocolate) may improve cognitive function ${ }^{29}$ and reduce fatigue ${ }^{30}$. Clinical observation also revealed that cocoa may beneficially modulate $\operatorname{mood}^{29}$. The biological activities/properties of cocoa are modulated by the bioavailability of its bioactive constituents which include polyphenols ${ }^{20,21}$. Cocoa flavanols have been shown to possess many biological activities including strong anti-inflammatory activities in vitro. Cocoa flavanols are capable of modulating TNF- $\alpha$ and IL-1 $\beta$ in peripheral blood mononuclear cells $(\mathrm{PBMCs})^{31,32}$. Cocoa proanthocyanidin monomers and dimmers can also inhibit nuclear factor кB (NF-кB) activation $^{33}$. Based on these observations and the emerging significance of inflammation in the pathogenesis of MDD, the current study tests the efficacy of a specific flavanol rich cocoa preparation (FRP) in attenuating depression in the repeated social defeat stress (RSDS) mouse model of depression.

\section{Materials and methods}

Commercially available FRP CocoaVia ${ }^{\circledR}$ was purchased from Cocoavia.com (Lot number 144533, MARS International). The cocoa extract was chemically profiled for polyphenols (see below) and archived in compliance with $\mathrm{NCCIH}$ Product Integrity guidelines. Phenolic standards including gallic acid (GA), caffeic acid (CA), trans-pcoumaric acid $(p-C A)$, catechin $((+)-C)$, epicatechin ((-)-EC), dihydrocoumaric acid (diHCA), 3-(3,4-dihydroxyphenyl)propionic acid (3,4-diHPPA), 3,4-dihydroxybenzoic acid (3,4-diHBA), hippuric acid (HA), homovanillic acid (HVA), 3-hydroxybenzoic acid (3HBA), 4-hydroxybenzoic acid (4-HBA), 3hydroxyphenylacetic acid (3-HPAA), 3-(3-hydroxyphenyl)propionic acid (3-HPPA), vanillic acid (VA), and trans-cinnamic acid- $\mathrm{d}_{7}$ (internal standard) were purchased from Sigma-Aldrich (St. Louis, MO, USA); 3,4dihydroxyphenylacetic acid (3,4-diHPAA) and ferulic acid (FA) were from ChromaDex Inc. (Irvine, CA); 5-(4hydroxyphenyl)valeric acid (4-HPVA) was from Alfa
Aesar (UK). Acids and solvents (all HPLC Grade) including glacial acetic acid (AA), formic acid (FA), acetonitrile $(\mathrm{ACN})$, methanol $(\mathrm{MeOH})$ and ethyl acetate were obtained from Fisher Scientific (Pittsburgh, PA, USA), and Pierce ${ }^{\mathrm{TM}}$ LC-MS water from Thermo Fisher Scientific (Waltham, MA, USA).

\section{Animals}

All C57BL/6 mice were purchased from the Jackson Laboratory (Bar Harbor, ME). Retired breeder CD-1 mice were purchased from Charles River Laboratory (Wilmington, MA). All animals had access to regular chow ad lib and were maintained on a 12:12-h light/dark cycle with lights on at $07: 00 \mathrm{~h}$ in a temperature-controlled $\left(20 \pm 2{ }^{\circ} \mathrm{C}\right)$ vivarium and all procedures were approved by the Mount Sinai Institutional Animal Care and Use Committee.

\section{Treatment}

For the dose-finding study, 8-week-old male C57BL/6 mice were randomly allocated into experimental groups and fed a polyphenol-free diet for 10 days and then treated with vehicle, $8,40,200$, and $500 \mathrm{mg} / \mathrm{kg}$ body weight $(\mathrm{BW}) /$ day FRP, delivered through their drinking water for 2 weeks. Mice were then challenged with intraperitoneal (i.p.) injection of $0.4 \mathrm{mg} / \mathrm{kg}$-BW lipopolysaccharide (LPS) and plasma levels of IL- 6 were measured $6 \mathrm{~h}$ post injection. For the bioavailability study, 6-weekold male C57BL/6 mice were fed with polyphenol-free diet for 10 days and then treated with a vehicle, $40 \mathrm{mg} / \mathrm{kg}$ $\mathrm{BW} /$ day or $200 \mathrm{mg} / \mathrm{kg}-\mathrm{BW} /$ day FRP for 2 weeks before sacrificing. For the in vivo efficacy study, 6-week-old male C57BL/6 mice were fed a polyphenol-free diet for 10 days and the mice were then randomly grouped into two: one group received regular drinking water and the other group received FRP-adulterated water $(40 \mathrm{mg} / \mathrm{kg}-\mathrm{BW} /$ day), starting 2 weeks prior to RSDS and throughout RSDS and social interaction (SI) testing.

\section{Quantitative analysis of the flavanol rich cocoa preparation}

For LC-DAD-MS analysis, a Hewlett Packard Agilent 1100 Series LC/MS (Agilent Technologies, Waldbronn, Germany) equipped with autosampler, quaternary pump system, DAD detector, degasser, MSD trap with an electrospray ion (ESI) source was employed. Data acquisition and processing was achieved using HP ChemStation, Bruker Daltonics and DataAnalysis software (Agilent, ver 4.1). Chromatographic separation was performed on a Polaris amide-C18 column, $250 \times 4.6 \mathrm{~mm}$, 5um (Varian Inc.) with a binary mobile phase containing solvent $\mathrm{A}$ $(0.1 \% \mathrm{FA}$ in water) and $\mathrm{B}(0.1 \% \mathrm{FA}$ in $\mathrm{ACN})$. Elution gradient started at $10 \% \mathrm{~B}$ and linearly increased to $20 \%$ in $20 \mathrm{~min}$; during $20-30 \mathrm{~min}$, linearly increase to $30 \% \mathrm{~B}$; during $30-40 \mathrm{~min}$, isocratic elution at $30 \% \mathrm{~B}$ with a flow rate of $1.0 \mathrm{~mL} / \mathrm{min}$. The UV detector was set to monitor 
at 254 (for hippuric acid), 280 (for phenolic acid \& flavanols), 370 (for flavonols), and 520 (for anthocyanidins) nm. Mass spectral data acquisition was achieved under positive polarity $(\mathrm{ESI}+)$ with a needle voltage at $3.5 \mathrm{kV}$ and scanned from $\mathrm{m} / z 100$ to 1200 . Nitrogen was used as dry gas at a flow rate of $12 \mathrm{~L} / \mathrm{min}$ and capillary temperature was at $350^{\circ} \mathrm{C}$. Nitrogen was used as nebulizer gas at $60 \mathrm{psi}$, and helium as collision gas.

\section{Plasma bioavailability}

Eight-week-old male C57BL/6 mice were fed a polyphenol-free diet for 10 days and then treated with a vehicle, $40 \mathrm{mg} / \mathrm{kg}-\mathrm{BW} /$ day or $200 \mathrm{mg} / \mathrm{kg}-\mathrm{BW} /$ day FRP for 2 weeks. On the day of sacrifice, mice were gavaged with a daily dose of the FRP and blood samples were collected one hour (for phase II polyphenol conjugates detection) or $6 \mathrm{~h}$ (for phenolic acids detection) following the gavage. Plasma was collected by centrifugation at $1500 \times g$ for $10 \mathrm{~min}$ and formic acid was added to the plasma to a final concentration of $0.2 \%$. Samples were snap frozen and stored at $-80^{\circ} \mathrm{C}$ until further analysis.

\section{Analysis of Phase II metabolites of polyphenol conjugates} Sample preparation Plasma samples were previously stored in $-80{ }^{\circ} \mathrm{C}$ freezer and transferred to $-20^{\circ} \mathrm{C} 24 \mathrm{~h}$ prior to analysis, thawed on ice, and conditioned to room temperature before processing. A thawed aliquot of plasma $(100 \mu \mathrm{L})$ was added to $500 \mu \mathrm{L}$ methanol containing $2 \%$ acetic acid and vortexed for $30 \mathrm{~s}$. The sample was allowed to stand still for $5 \mathrm{~min}$ for complete protein precipitation, followed by centrifugation at $16,000 \times g$ for $10 \mathrm{~min}$. The precipitate was re-mixed with $200 \mu \mathrm{L}$ of acidified methanol and extracted one more time. The pooled supernatant was then transferred to a glass vial and dried under a gentle stream of nitrogen. The residues were then reconstituted in $200 \mu \mathrm{L} 20 \%$ acetonitrile in water containing $0.1 \%$ formic acid. The reconstituted sample was centrifuged at $16000 \times g$ for $10 \mathrm{~min}$ and $10 \mu \mathrm{L}$ of the supernatant was injected into the LC-MS/MS system.

Phase II metabolites analysis Phase II metabolites from plasma samples were analyzed using an Agilent 1290 Infinity II UPLC (Agilent Technology, Atlanta, GA, USA) system interfaced with an Agilent 6400 Triple Quadrupole Mass Spectrometer (LC-QqQ/MS) with an ESI source. A Waters ACQUITY UPLC BEH C18 Column $(2.1 \times 50 \mathrm{~mm}$ with $1.7 \mu \mathrm{m}$ particle size) was used with a thermostat set at $30{ }^{\circ} \mathrm{C}$. The binary mobile phase consisted of $0.1 \%$ formic acid in water (solvent $\mathrm{A}$ ) and $0.1 \%$ formic acid in acetonitrile (solvent $\mathrm{B}$ ). The flow rate was set to $0.4 \mathrm{~mL} /$ min. The gradient conditions used were $2 \% \mathrm{~B}$ at $0 \mathrm{~min}, 5 \%$ $\mathrm{B}$ at $6 \mathrm{~min}, 25 \% \mathrm{~B}$ at $10 \mathrm{~min}, 95 \% \mathrm{~B}$ at $12 \mathrm{~min}$, and back to $2 \% \mathrm{~B}$ at $13 \mathrm{~min}$ with $2 \mathrm{~min}$ post-run equilibration. The MS was operated with positive polarity under multiple reaction monitoring (MRM) mode. The MRM transition for $(+)$-catechin (C) and (-)-epicatechin (EC) was $291 \rightarrow$ 139, the transition for $\mathrm{C}^{\prime}$-O-glucuronide (C-glucur) and EC 3'-O-glucuronide (EC-glucur) was $467 \rightarrow 291$, and the transition for Methyl O-C-glucuronide (Me-C-glucur) and Methyl O-EC-glucuronide (Me-EC-glucur) was $481 \rightarrow 305$. The fragment voltage used was set at $106 \mathrm{~V}$, the collision energy at $12 \mathrm{eV}$ and the cell accelerator voltage at $4 \mathrm{~V}$. The ESI conditions were set with the nebulizer pressure at $30 \mathrm{psi}$, the capillary voltage at $3500 \mathrm{~V}$ and the nozzle voltage at $1000 \mathrm{~V}$, the drying gas temperature at $350{ }^{\circ} \mathrm{C}$ with a flow rate of $12 \mathrm{~L} / \mathrm{min}$, and the sheath gas temperature at $350{ }^{\circ} \mathrm{C}$ with a flow rate of $12 \mathrm{~L} / \mathrm{min}$. Glucuronides of $\mathrm{C}$ and $\mathrm{EC}$ was estimated using a calibration curve constructed with an authentic quercetin 3-O-glucuronide (Quer-gluc) standard and corrected by a molecular weight ratio (metabolite/Quergluc ratio). Methylated $\mathrm{C} / \mathrm{EC}$ was quantified using the same calibration curve for $\mathrm{C} / \mathrm{EC}$.

\section{Phenolic acid metabolite analysis}

Sample preparation Plasma samples were previously stored at $-80^{\circ} \mathrm{C}$ and transferred to $-20^{\circ} \mathrm{C} 24 \mathrm{~h}$ prior to analysis. Immediately before processing, samples were thawed on ice and then conditioned to room temperature. An internal standard (I.S.), trans-cinnamic acid ( $\left.\mathrm{d}_{7}\right)(2 \mu \mathrm{g} /$ $\mathrm{mL}$ ) was mixed with $0.4 \mathrm{M} \mathrm{NaH}_{2} \mathrm{PO}_{4}$ buffer ( $\mathrm{pH}$ 5.0) and a $300-\mu \mathrm{L}$ aliquot containing $5 \mu \mathrm{L}$ of I.S. was added to $100 \mu \mathrm{L}$ of thawed plasma. The mixture was then incubated with 300 of $\mathrm{NaH}_{2} \mathrm{PO}_{4}$ buffer and $50 \mu \mathrm{L}$ of the solution of $\beta$-glucuronidase $(2000 \mathrm{U})$ in contamination with sulfatase (diluted in $\mathrm{NaH}_{2} \mathrm{PO}_{4}$ buffer). For plasma samples with volume less than $100 \mu \mathrm{L}$, enzymatic digestion was carried out directly in the original Eppendorf tube as aforementioned. All samples were incubated at $37^{\circ} \mathrm{C}$ for $45 \mathrm{~min}$ after purging with nitrogen. Enzymatic reaction was stopped by adding $500 \mu \mathrm{L}$ of ethyl acetate. The mixture was vortexed vigorously for $1 \mathrm{~min}$, followed by centrifugation at $3000 \times g$ for $5 \mathrm{~min}$. The upper organic phase was transferred to a 1-dram glass vial. After two more extractions with ethyl acetate $(500 \mu \mathrm{L})$, the pooled supernatant was mixed with $10 \mu \mathrm{L}$ of $2 \%$ ascorbic acid in methanol and dried under a gentle stream of nitrogen. The residue was reconstituted in $100 \mu \mathrm{L}$ of $45 \%$ methanol containing $0.1 \%$ formic acid and centrifuged at $16,000 \times g$ for $10 \mathrm{~min}$.

Phenolic acids analysis The analyses of $\mathrm{C}, \mathrm{EC}$, and phenolic acid metabolites (PAMs) were carried out on an Agilent 1290 Infinity II UPLC system interfaced with an Agilent 6470 Triple Quadrupole Mass Spectrometer with an ESI source (Agilent Technology, Palo Alto, CA, USA). 
For each sample extract, $5 \mu \mathrm{L}$ was injected into an UPLC$\mathrm{QqQ} / \mathrm{MS}$ system for analysis using the method developed under dynamic multiple reaction monitoring (dMRM) mode.

\section{Assessment of IL- 6 expression in vitro using periphery blood mononuclear cells (PBMCs)}

PBMCs from 2-month-old mice were isolated using the Ficoll-Paque density-gradient centrifugation method. Specifically, whole blood was mixed with complete RPMI media and laid over the Ficoll-Paque Plus (GE Healthcare, Sweden), centrifuged at $2200 \mathrm{RPM}$ for $15 \mathrm{~min}$. The buffy coat containing PBMCs were isolated, washed once with BEP (0.5\% BSA, 2 mM EDTA in PBS) and plated out in a 24-well plate at $5 \times 10^{5} /$ well in culture medium RPMI-1640 (Sigma) supplemented with $20 \%$ horse serum, 10\% FBS, $2.05 \mathrm{mM}$ L-glutamine, $25 \mathrm{mM}$ Hepes, and $100 \mathrm{U} / \mathrm{ml}$ penicillin/streptomycin. PBMCs were treated with phenolic acids for $16 \mathrm{~h}$ and challenged with $7.5 \mu \mathrm{g} / \mathrm{ml}$ LPS. Supernatant was collected by centrifugation following $16 \mathrm{~h}$ of LPS treatment and the levels of cytokines were measured using the Multiplex MAP mouse cytokine/ chemokine Panel (EMD Millipore) following the manufacturer's instruction.

\section{Behavioral testing RSDS}

RSDS was performed as previously described ${ }^{34,35}$. CD-1 mice were screened for aggressive characteristics prior to the start of social defeat experiments based on previously described criteria $^{35}$, and housed in the social defeat cage $(26.7 \mathrm{w} \times 48.3 \mathrm{~d} \times 15.2 \mathrm{~h} \mathrm{~cm}$; Allentown Inc) $24 \mathrm{~h}$ prior to the start of defeats on one side of a clear, perforated Plexiglass divider $(0.6 \times 45.7 \times 15.2 \mathrm{~cm}$; Nationwide
Plastics). Briefly, mice subjected to RSDS were exposed to a novel CD-1 aggressor mouse for $10 \mathrm{~min}$ once per day, over 10 consecutive days. Following the 10-min interaction, the experimental mice were removed to the opposite side of the social defeat cage and sensory contact during the following 24-h period was allowed. Mice were returned to a single house following the last defeat and before the social avoidance test.

\section{Social interaction test (social avoidance test)}

Social interaction (SI) testing was performed as previously described $^{35}$. All SI testings were performed under red-light conditions. Mice were placed in a novel interaction open-field arena custom-crafted from opaque Plexiglas $(42 \times 42 \times 42 \mathrm{~cm}$; Nationwide Plastics $)$ with a small animal cage placed at one end. Their movements were then automatically monitored and recorded (Ethovision 3.0; Noldus Information Technology) for $2.5 \mathrm{~min}$ in the absence (target absent phase) of a novel CD-1 mouse. This phase is used to determine baseline exploratory behavior. We then immediately measured $2.5 \mathrm{~min}$ of exploratory behavior in the presence of a caged CD-1 mouse (target present phase), again recording the total distance traveled and the duration of time spent in the interaction and corner zones. SI behavior was then calculated as total time spent in each zone, or as a ratio of the time spent in the interaction with the target present divided by the time spent in the interaction zone with the target absent. All mice with a ratio above 1.0 were classified as resilient whereas below 1.0 were classified as susceptible.

\section{Overall statistics}

All values are expressed as mean and standard error of the mean (s.e.m.). Unpaired two-tailed Student's $t$-tests

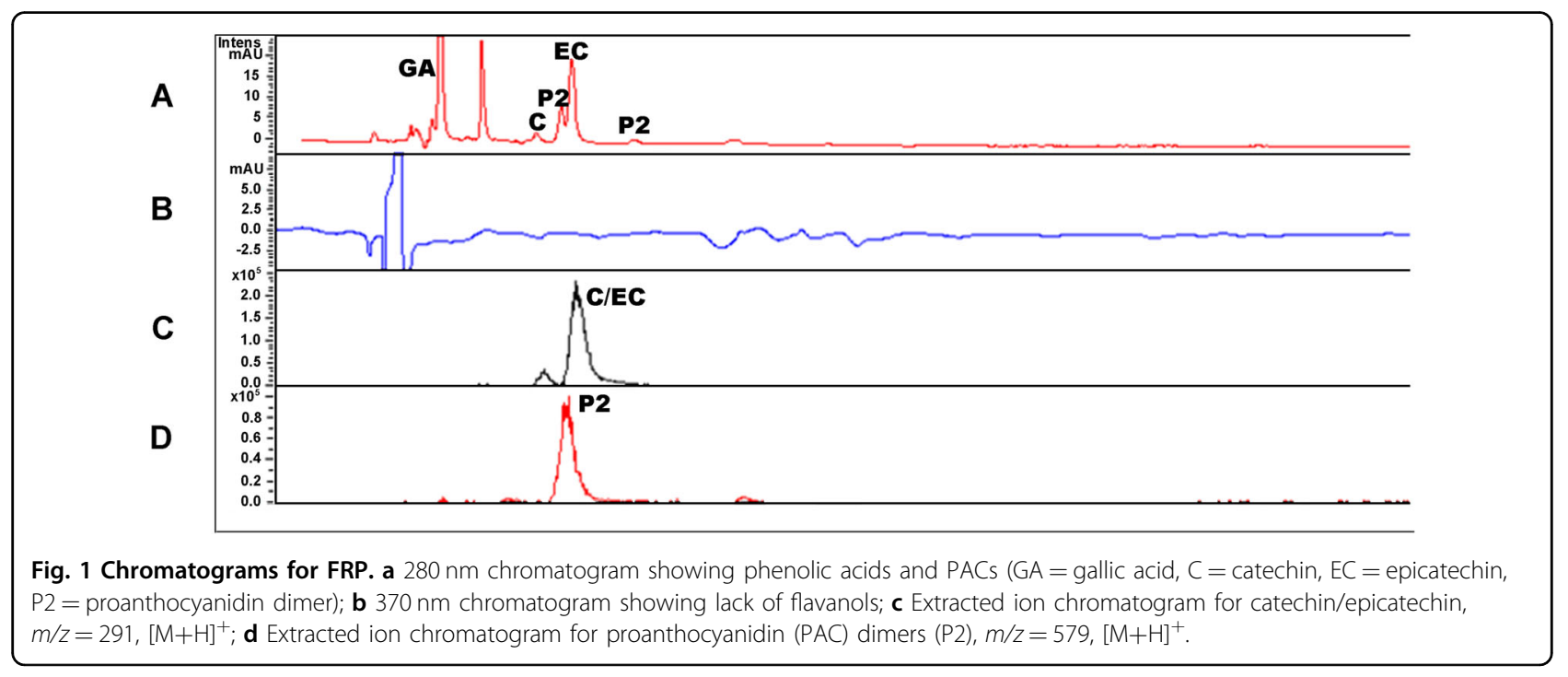


with Welch's correction were used. In all studies, outliers (2 SD from the mean) were excluded and the null hypothesis was rejected at the 0.05 level. All statistical analyses were performed using Prism Stat program (GraphPad Software, Inc.).

\section{Results}

\section{Flavanol identification and quantification}

Cocoa contains a high content of the flavonoids $(-)$-epicatechin, $(+)$-catechin and their dimers, proanthocyanidin B1 and B2, and the composition largely depends on the type of cocoa beans and the extraction methods. We measured the content of flavanols in the FRP under positive ionization mode with UV detection. The representative UV chromatograms $(280 \mathrm{~nm})$ and MS chromatograms of FRP polyphenols are illustrated in Fig. 1. On the basis of UV and MS spectral data and by comparison to the retention time of the authentic standards, we were able to detect catechin, epicatechin, and proanthocyanidin (PAC) dimers. The FRP contains approximately $1.92 \%$ of catechin, $7.97 \%$ of epicatechin, $6.97 \%$ of PAC dimers and $5.62 \%$ of gallic acid. We did not detect a measurable amount of flavanols, galloylated PAC dimers/ trimers or PAC trimers/tetramers (Table 1).

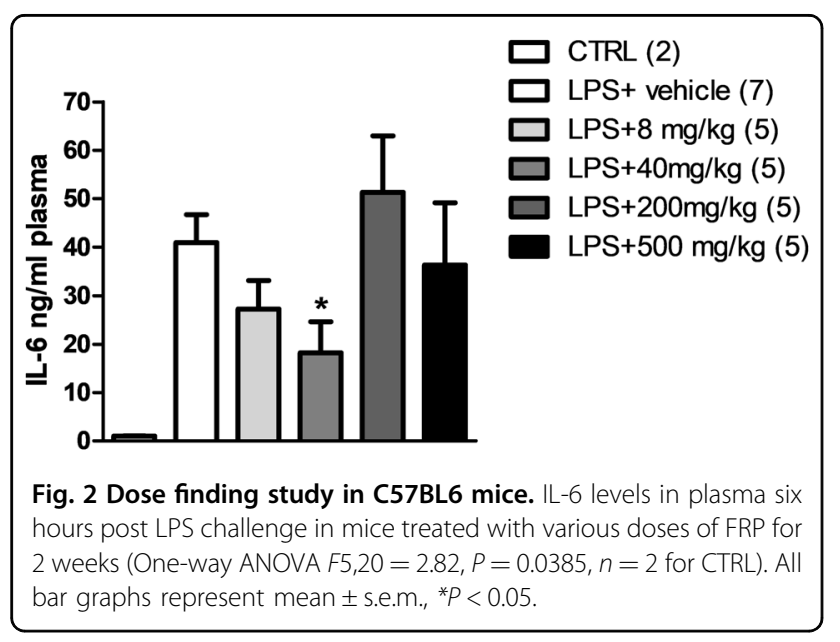

Table 1 Quantitative analysis of FRP.

\begin{tabular}{ll}
\hline Compound & mg/g (\%) \\
\hline Catechin & $19.2 \pm 0.176(1.92)$ \\
Epicatechin & $79.7 \pm 0.315(7.97)$ \\
P2 isomers & $69.7 \pm 0.608(6.97)$ \\
Gallic acid & $56.2 \pm 0.658(5.62)$ \\
\hline
\end{tabular}

Chemical composition of FRP was quantified using HPLC/UV/MS: P2 = proanthocyanidin dimer.

\section{In vivo dose finding studies for FRP}

In this study, we chose to target peripheral inflammation. We initiated dose response studies in mice to determine testing dosages for preclinical efficacy as a potential intervention for stress-induced depression. C57BL/6 wild-type mice were treated with FRP (doses ranging from $8 \mathrm{mg}$ to $500 \mathrm{mg} / \mathrm{kg}$-BW/day) for 2 weeks to simulate long-term administration. Mice were then challenged with intraperitoneal (i.p.) injection of $0.4 \mathrm{mg} / \mathrm{kg}$ LPS and plasma levels of IL- 6 were measured $6 \mathrm{~h}$ postinjection. We found that pretreatment with lower doses of FRP led to a dose-dependent suppression of IL-6 production in plasma and that the group treated with $40 \mathrm{mg} /$ $\mathrm{kg}-\mathrm{BW} /$ day showed significant reduction compared to the vehicle-treated group. However, treatment with 200 and $500 \mathrm{mg} / \mathrm{kg}-\mathrm{BW} /$ day did not show any effect in reducing LPS-mediated peripheral increase of IL-6 (Fig. 2).

\section{Plasma bioavailability studies}

Based on the observation that $40 \mathrm{mg} / \mathrm{kg}$-BW/day significantly reduced LPS-induced IL-6 production in plasma while neither 200 nor $500 \mathrm{mg} / \mathrm{kg}$-BW/day had effects, we reasoned that the differences may be due to the cumulative effect of polyphenol metabolites in the plasma following oral administration. To investigate qualitatively as well quantitatively the differences between 40 and $200 \mathrm{mg} / \mathrm{kg}$-BW/day dosage, we initiated a bioavailability study. The majority of orally consumed dietary polyphenols, including those in FRP, are extensively metabolized during gastrointestinal absorption and/or postabsorptive xenobiotic metabolism, converting them into phase II polyphenol conjugate metabolites. In addition, polyphenols can also be metabolized by intestinal bacteria into phenolic acids ${ }^{36,37}$. Thus, orally consumed polyphenols are typically bioavailable in vivo as phenolic metabolites (polyphenol conjugate metabolites and phenolic acids). Previously we have shown that polyphenols from a flavanol-rich grape seed polyphenol extract (GSPE) were metabolized to phase II polyphenol conjugates fairly rapidly and peaked about one hour following oral gavaging $^{26}$. On the other hand, it took roughly $6 \mathrm{~h}$ for the microbiome-derived phenolic acids to peak ${ }^{38}$. One group of mice was sacrificed $60 \mathrm{~min}$ following the final dose of gavaging and plasma was evaluated for phase II conjugates. Not surprisingly, we found a dose-dependent increase of phase II polyphenol metabolites in the forms of glucuronidated or methylated and glucuronidated catechin/epicatechin (Table 2). The second group of mice was sacrificed $6 \mathrm{~h}$ after the final dosing and plasma was evaluated for phenolic acid metabolites (PAMs). We found a dose-dependent increase of gallic acid, 3,4-dihydroxybenoic acid, 5-(4-hydroxyphenyl) valeric acid, and hippuric acid (Table 3). Interestingly, we also found a significant increase of trans- $\rho$-coumaric acid (4-HCA), 
Table 2 Measurements of phase II polyphenol conjugates in plasma following FRP treatment.

\begin{tabular}{|c|c|c|c|c|c|c|}
\hline \multirow{2}{*}{$\begin{array}{l}\text { Phase II } \\
\text { Polyphenol }\end{array}$} & \multicolumn{2}{|l|}{ Vehicle CTRL } & \multicolumn{2}{|l|}{$40 \mathrm{mg} / \mathrm{kg} / \mathrm{day}$} & \multicolumn{2}{|l|}{$200 \mathrm{mg} / \mathrm{kg} /$ day } \\
\hline & Average (nM) & St dev & Average (nM) & St dev & Average (nM) & St dev \\
\hline Me-C-Gluc & N.D. & & 0.70 & 0.23 & 1.81 & 1.09 \\
\hline Me-C/EC-Gluc* & N.D. & & 1.39 & 0.81 & 3.28 & 1.69 \\
\hline Me-EC-Gluc & N.D. & & 1.66 & 0.92 & 4.42 & 2.55 \\
\hline C-Gluc & N.D. & & 2.29 & 2.88 & 11.37 & 3.06 \\
\hline EC-Gluc & N.D. & & 3.47 & 3.06 & 16.97 & 4.87 \\
\hline C & N.D. & & 5.34 & 2.28 & 15.40 & 3.81 \\
\hline EC & N.D. & & 10.27 & 7.41 & 35.36 & 12.69 \\
\hline
\end{tabular}

Plasma levels of $3^{\prime}$-O-methyl-catechin-5-O- 3 -glucuronide (Me-C-Gluc), methyl-catechin/epicatechin-glucuronide (Me-C/EC-Gluc), 3'-O-methyl-epicatechin-5-O$\beta$-glucuronide (Me-EC-Gluc), catechin-glucuronide (C-Gluc), epicatechin-glucuronide (EC-Gluc), catechin (C), and epicatechin (EC) measured $1 \mathrm{~h}$ following the last dosing in mice treated with $40 \mathrm{mg} / \mathrm{kg} /$ day or $200 \mathrm{mg} / \mathrm{kg} /$ day of FRP for 2 weeks, $n=5$ for vehicle control, $n=7$ for treatment group.

Table 3 Measurements of phenolic acids in plasma following FRP treatment.

\begin{tabular}{|c|c|c|c|c|c|c|}
\hline \multirow[t]{2}{*}{ Phenolic acids } & \multicolumn{2}{|l|}{ Vehicle CTRL } & \multicolumn{2}{|l|}{$40 \mathrm{mg} / \mathrm{kg} / \mathrm{day}$} & \multicolumn{2}{|l|}{$200 \mathrm{mg} / \mathrm{kg} /$ day } \\
\hline & Average (nM) & St dev & Average (nM) & St dev & Average (nM) & St dev \\
\hline $\mathrm{GA}^{\mathrm{a}}$ & 38.9 & 12.4 & 72.3 & 23.5 & 126.4 & 31.8 \\
\hline 3,4-diHBA ${ }^{a}$ & 14.0 & 11.7 & 185.9 & 47.3 & 247.8 & 73.4 \\
\hline 3,4-diHPPA & 459.2 & 59.2 & 566.6 & 139.0 & 525.8 & 133.8 \\
\hline 4-HBA & 4174.6 & 191.6 & 4335.2 & 253.7 & 4152.1 & 184.9 \\
\hline$H A^{a}$ & 10.5 & 14.4 & 125.7 & 65.0 & 641.7 & 566.6 \\
\hline$V A^{b}$ & 555.2 & 63.1 & 667.4 & 99.8 & 614.9 & 40.8 \\
\hline $3-\mathrm{HBA}$ & 579.4 & 10.3 & 578.7 & 12.4 & 587.8 & 12.0 \\
\hline $3-\mathrm{HPPA}^{\mathrm{b}}$ & 1046.8 & 32.8 & 1228.7 & 125.1 & 1039.8 & 51.4 \\
\hline$H V A^{b}$ & 5161.8 & 470.9 & 8952.9 & 1950.4 & 7045.7 & 1048.6 \\
\hline $\mathrm{diHCA}$ & 205123.3 & 5945.6 & 195755.1 & 6280.6 & 174706.9 & 15465.6 \\
\hline $4-H C A^{b}$ & 1535.3 & 224.6 & 3456.7 & 1119.1 & 2670.5 & 738.4 \\
\hline 3-HPPA & 468.5 & 4.6 & 505.0 & 60.8 & 513.1 & 18.8 \\
\hline FA & 308.8 & 9.5 & 360.1 & 40.3 & 352.8 & 21.9 \\
\hline 4-HPVA & 0.0 & 0.0 & Trace & 0.0 & 31.4 & 11.4 \\
\hline
\end{tabular}

Plasma levels phenolic acids measured $6 \mathrm{~h}$ following the last dosing in mice treated with vehicle, $40 \mathrm{mg} / \mathrm{kg} / \mathrm{day}$ or $200 \mathrm{mg} / \mathrm{kg} / \mathrm{day}$ of FRP for $2 \mathrm{weeks}$.

${ }^{\text {aP }}$ henolic content significantly higher in the $200 \mathrm{mg} / \mathrm{kg} /$ day group compared to $40 \mathrm{mg} / \mathrm{kg} /$ day group.

${ }^{\mathrm{b}}$ Phenolic content significantly higher in the $40 \mathrm{mg} / \mathrm{kg} /$ day group compared to $200 \mathrm{mg} / \mathrm{kg} /$ day group, $n=5$ for vehicle control, $n=7-8$ for treatment group.

homovanillic acid (HVA), 3-hydroxyphenylacetic acid (3HPAA), and vanillic acid (VA) in the $40 \mathrm{mg} / \mathrm{kg}-\mathrm{BW} /$ day compared to the vehicle control group and the $200 \mathrm{mg} /$ $\mathrm{kg}$-BW/day group (Fig. 3). We did not find significant changes in the amount of 3-hydroxybenzoic acid, dihydrocoumaric acid, 4-hydroxybenzoic acid, 3-(3-hydroxyphenyl) propionic acid, ferulic acid, or 3,4- dihydroxyphenylacetic acid (Table 3), suggesting these phenolic acids are very unlikely to be derived from FRP consumption, rather endogenously available. It is also possible that the production and excretion of these phenolic acid metabolites reach a dynamic equilibrium in the body and FRP consumption does not significantly affect their levels. 


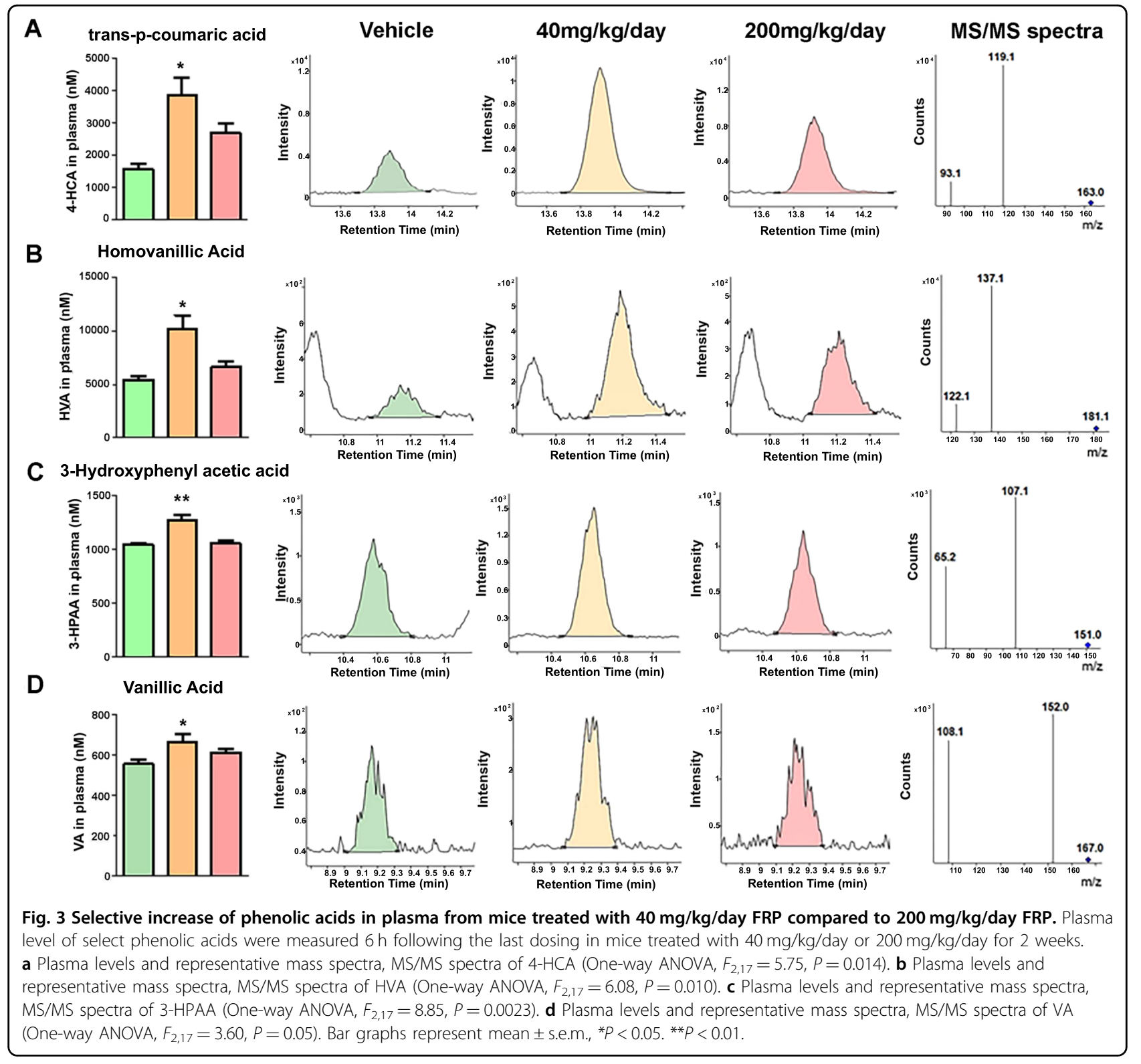

In vitro testing of the anti-inflammatory activity of 4-HCA, HVA, 3-HPAA, and VA

The bioavailability data showed that the level of 4-HCA, HVA, 3-HPAA, and VA were significantly higher in the 40-mg/kg-BW/day group compared to the vehicle-treated group, while $200 \mathrm{mg} / \mathrm{kg}-\mathrm{BW} /$ day had similar amounts as the vehicle-treated group. The dose finding studies demonstrated that $40 \mathrm{mg} / \mathrm{kg} /$ day FRP can significantly reduce the LPS-mediated peripheral IL-6 induction, while $200 \mathrm{mg} / \mathrm{kg} /$ day treatment had no effect, with similar levels of IL- 6 relative to the vehicle treatment. We hypothesized that these phenolic acids might be, in part, responsible for the attenuation of LPS-induced IL-6. To test this hypothesis, we treated peripheral blood mononuclear cells
(PBMCs) isolated from wild-type C57BL6 mice with these four compounds either alone or in combination for $16 \mathrm{~h}$. The concentrations we used were the same concentrations as identified in the plasma following $40 \mathrm{mg} / \mathrm{kg} /$ day treatment: $4 \mu \mathrm{M}$ 4-HCA, $10 \mu \mathrm{M}$ HVA, $1.25 \mu \mathrm{M} 3$-HPAA, and $0.7 \mu \mathrm{M}$ VA. Following the treatment, PBMCs culture were challenged with $1 \mu \mathrm{g} / \mathrm{ml}$ LPS for $16 \mathrm{~h}$ and cytokines were measured using a Multiplex MAP mouse cytokine/ chemokine panel. Interestingly, we found that when pretreated with a single compound, only HVA significantly reduced the production of IL-6, while the other three phenolic acids had no effect (Fig. 4a). However, when 4 phenolic acids were applied together, we observed a synergistic effect and the level of IL- 6 was significantly 

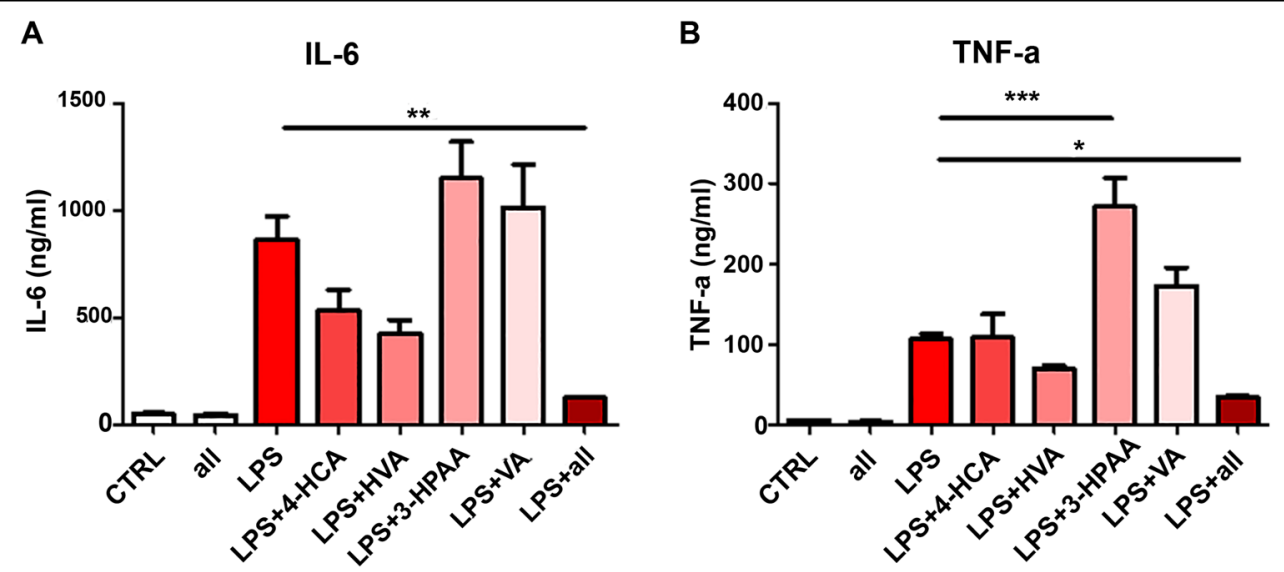

Fig. 4 In vitro testing of phenolic acids in attenuating LPS-induced production of inflammatory cytokines. Mouse PBMCS were treated with either individual phenolic acid or the combination of all 4 phenolic acids that found to be significantly higher in the $40 \mathrm{mg} / \mathrm{kg} / \mathrm{day} \mathrm{group}$ for $16 \mathrm{~h}$ and stimulated with LPS for 16h. Cytokine content in the supernatant was measured by multiplex ELISA. a The level of IL-6 (One-way ANOVA, $F_{7,31}=$ $15.95, P<0.0001, n=4$ per culture conditions) and $\mathbf{b}$ the level of TNF-a (One-way ANOVA, $F_{7,31}=28.46, P<0.0001, n=4$ per culture conditions). Bar graphs represent mean \pm s.e.m., ${ }^{*} P<0.05,{ }^{* *} P<0.01,{ }^{* * *} P<0.001$.

lower compared to the culture that was treated with HVA alone (Fig. 4a). Similarly, we found none of the phenolic acids significantly reduced TNF- $\alpha$ production when applied alone. 3-HPAA treatment significantly increased TNF- $\alpha$ (Fig. 4b). However, when the 4 phenolic acids were applied together, there was a significant reduction of TNF- $\alpha$ (Fig. 4b). Cell viability assessment by Trypan Blue staining showed that the cells were all viable following the treatment and following LPS stimulation.

\section{Treatment with FRP promotes resilience to RSDS-induced social avoidance behavior}

Our dose-finding studies demonstrate that $40 \mathrm{mg} / \mathrm{kg}$ BW/day FRP can attenuate LPS-induced IL-6 production. Based upon these results, as well as in vitro evidence demonstrating that a combination of phenolic acids can attenuate LPS-induced inflammatory cytokine production, we conducted a proof of concept study to evaluate the potential role of FRP using the well-established RSDS model. Mice were fed on a polyphenol-free diet for 10 days and were then treated with FRP or vehicle for 2 weeks prior to and throughout RSDS. Following 10 days of RSDS, mice were subjected to the social avoidance test (Fig. 5a). We found that treatment with FRP greatly increased the proportion of mice resilient to the stress as indicated by normal social interactions, compared to the vehicle-treated animals. Overall, $\sim 77 \%$ of mice receiving FRP showed resilient behavioral phenotypes, whereas only $\sim 46 \%$ were resilient in the control groups (Fig. 5b, c).

\section{Discussion}

MDD has become one of the leading causes of disability worldwide. Based on the data obtained from National Institute of Mental Health, it is estimated that over 15 million people experience at least one episode of major depression each year in the United States alone. Conventional anti-depressant treatments mainly target the monoaminergic system and $~ 50 \%$ of the patients do not fully respond to the currently available treatment, which may reflect the heterogeneity of the pathogenesis of the disorder. Since the identification of the role of inflammation in the pathophysiology of depression in a subset of hepatitis patients treated with interferon- $\alpha^{39}$, there is a growing interest in the field of psychoneuroimmunology to identify inflammatory molecules/cells as potential therapeutic targets for depression. Among these, IL-6 was the most consistently observed inflammatory cytokine that is closely associated with MDD ${ }^{14-16}$. IL-6 has emerged as a potential promising therapeutic target for MDD.

Natural products have a history of being the source for many of the active ingredients in medications, and almost half of the drugs approved since 1994 are derived or inspired from natural products ${ }^{40,41}$. In recent years, cocoa products, specifically cocoa polyphenols, have received growing interest due to epidemiological observation that people consuming cocoa products such as chocolate reported either improvement in mood or attenuation of negative $\operatorname{mood}^{29}$. The benefits of chocolate have been in part, attributed to cocoa flavanols ${ }^{42,43}$. Flavanols are known to be able reduce the production of proinflammatory molecules and to directly or indirectly impact the signaling transduction pathways associated with inflammation ${ }^{31-33}$. This study was designed to test the potential contribution of cocoa flavanols in modulating depression-like behavior in a well-established mouse model of depression.

The dose study we first conducted and showed that higher dose FRP treatment $(200 \mathrm{mg} / \mathrm{kg} /$ day or $500 \mathrm{mg} / \mathrm{kg} /$ 


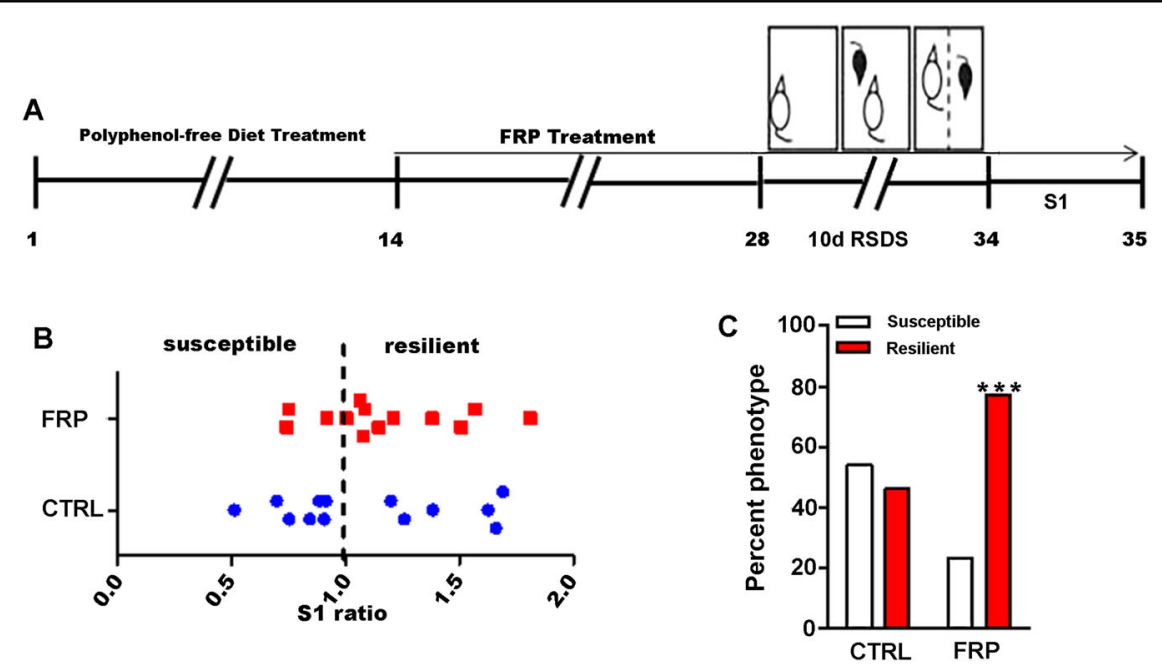

Fig. 5 Prophylactic effect FRP in promoting resilience to social stress. a Schematic design of the experiment. $\mathbf{b}$ Treatment with FRP increases the proportion of mice showing a resilient phenotype, as measured by social interaction ratio (c) percentage of susceptible and resilient mice following FRP and vehicle treatment. Bar graphs represent mean \pm s.e.m., Chi-square test, ${ }^{* * *} p<0.001, n=13$ per group.

day) had no effect on LPS-induced IL-6 production in mice while $40 \mathrm{mg} / \mathrm{kg} /$ day FRP treatment was very effective. This begs the question: what is the difference between the low and high dose treatment? Cocoa polyphenols, including flavanols, are extensively metabolized through xenobiotic and colonic microbiome-mediated metabolism following oral consumption, resulting in the generation of phenolic metabolites - polyphenol phase II metabolites and phenolic acids. Compared to the $40 \mathrm{mg} /$ $\mathrm{kg} /$ day and the $200 \mathrm{mg} / \mathrm{kg} /$ day treatment, we found that oral administration of FRP led to a dose-dependent accumulation of phase II polyphenol metabolites in plasma. There was also a dose-dependent accumulation of select phenolic acid metabolites in plasma. Unexpectedly, we found that the plasma contents of some of the phenolic acids, specifically 4-HCA, HVA, 3-HPAA, and VA, were lower in mice dosed with higher dose FRP.

In vitro studies showed that when applied together, these four compounds produced a synergistic effect in reducing IL- 6 and TNF- $\alpha$, suggesting that these phenolic acids might be partially responsible for the inhibition of IL-6 in mice challenged with LPS. This result is consistent with some of the human studies. In a randomized, controlled, double-blinded, balanced trial with 30 healthy adults designed to test the effect of cocoa flavanols on cognitive function, Scholey et al. $^{42}$ reported that subjects who consumed $520 \mathrm{mg}$ of cocoa flavanols benefited from "mental fatigue" while subjects who consumed $994 \mathrm{mg}$ of cocoa flavanols reported no benefits. These observations suggest that it is very important to consider the differential metabolism of cocoa polyphenols and the generation of various bioactive phase II polyphenol metabolites and phenolic acid metabolites when determining the dosages to be tested for human studies.

In this study, we detected at least 5 polyphenol conjugate metabolites and 14 phenolic acids with measurable amounts accumulating in plasma following oral administration. Here, we only examined four phenolic acids for their synergistic effect on anti-inflammation in vitro, and our results suggest that it is possible that some phenolic metabolites may counteract the positive effect of the other phenolic metabolites and that the overall benefits might be reduced due to potential "cancellation" effects. Future studies will continue to investigate the effect of the other phenolic metabolites on anti-inflammatory bioactivities and dissect the potential mechanisms.

As a proof of concept, we demonstrated that oral administration of FRP promoted resilience against social stress. Besides the anti-inflammatory activity, cocoa polyphenols are also known for their neuroprotective bioactivities. For example, epicatechin, one of the major components of FRP, can promote neurogenesis ${ }^{44}$. More pertinent to our current studies, epicatechin and its phenolic metabolites are effective scavengers of reactive oxygen species and exhibit potent antioxidant properties. Cocoa extract also contains significant level of methylxanthines including theobromine and caffeine, which can act on the central nervous system mainly through adenosine receptor signaling ${ }^{45}$. All of these bioactivities may contribute to the benefits of FRP in attenuating the development of depression-like phenotypes in the RSDS mouse model. Future studies will further dissect the potential contributions of each of these natural products in cocoa and their underlying mechanisms in modulating depression. 


\section{Acknowledgements}

Funding was provided by the P50 AT008661-01 from the National Center for Complementary and Integrative Health $(\mathrm{NCClH})$ and the Office of Dietary Supplements (ODS) and support from Altschul Foundation. In addition, J.W. and G.M.P. hold positions in the Research and Development unit of the Basic and Biomedical Research and Training Program, GRECC and G.M.P is a VA Senior Career Scientist at the James J. Peters Veterans Affairs Medical Center. We acknowledge that the contents of this manuscript are solely the responsibility of the authors and do not necessarily represent the views of the $\mathrm{NCCIH}$ or the U.S. Department of Veterans Affairs or the United States Government.

\section{Author details}

'Department of Neurology, Icahn School of Medicine at Mount Sinai, New York, NY 10029, USA. ${ }^{2}$ Geriatric Research, Education and Clinical Center, James J. Peters Veterans Affairs Medical Center, Bronx, New York, NY 10468, USA. ${ }^{3}$ New Use Agriculture \& Natural Plant Products Program, Department of Plant Biology, School of Environmental and Biological Sciences, Rutgers University, New Brunswick, NJ 08901, USA

\section{Conflict of interest}

The authors declare that they have no conflict of interest.

\section{Publisher's note}

Springer Nature remains neutral with regard to jurisdictional claims in published maps and institutional affiliations.

Received: 28 January 2020 Revised: 4 May 2020 Accepted: 15 May 2020 Published online: 09 June 2020

\section{References}

1. Kupfer, D. J., Frank, E. \& Phillips, M. L. Major depressive disorder: new clinical, neurobiological, and treatment perspectives. Lancet 379, 1045-1055 (2012).

2. Casacalenda, N., Perry, J. C. \& Looper, K. Remission in major depressive disorder: a comparison of pharmacotherapy, psychotherapy, and control conditions. Am. J. Psychiatry 159, 1354-1360 (2002).

3. Entsuah, A. R., Huang, H. \& Thase, M. E. Response and remission rates in different subpopulations with major depressive disorder administered venlafaxine, selective serotonin reuptake inhibitors, or placebo. J. Clin. Psychiatry 62, 869-877 (2001).

4. Palucha, A. \& Pilc, A. The involvement of glutamate in the pathophysiology of depression. Drug News Perspect. 18, 262-268 (2005).

5. Duman, R. S., Heninger, G. R. \& Nestler, E. J. A molecular and cellular theory of depression. Arch. Gen. Psychiatry 54, 597-606 (1997).

6. Kang, H. J. et al. Decreased expression of synapse-related genes and loss of synapses in major depressive disorder. Nat. Med. 18, 1413-1417 (2012).

7. Aston, C., Jiang, L. \& Sokolov, B. P. Transcriptional profiling reveals evidence for signaling and oligodendroglial abnormalities in the temporal cortex from patients with major depressive disorder. Mol. Psychiatry 10, 309-322 (2005).

8. Maes, M. Evidence for an immune response in major depression: a review and hypothesis. Prog. Neuropsychopharmacol. Biol. Psychiatry 19, 11-38 (1995).

9. Maes, M. et al. Increased serum IL-6 and IL-1 receptor antagonist concentrations in major depression and treatment resistant depression. Cytokine $\mathbf{9}$ 853-858 (1997).

10. Smith, R. S. The macrophage theory of depression. Med. Hypotheses $\mathbf{3 5}$ 298-306 (1991).

11. Stein, M., Keller, S. E. \& Schleifer, S. J. Stress and immunomodulation: the role of depression and neuroendocrine function. J. Immunol. 135, 827s-833s (1985).

12. Hodes, G. E., Kana, V., Menard, C., Merad, M. \& Russo, S. J. Neuroimmune mechanisms of depression. Nat. Neurosci. 18, 1386-1393 (2015).

13. Pfau, M. L., Menard, C. \& Russo, S. J. Inflammatory mediators in mood disorders: therapeutic opportunities. Annu. Rev. Pharmacol. Toxicol. 58, 411-428 (2017).

14. Howren, M. B., Lamkin, D. M. \& Suls, J. Associations of depression with Creactive protein, IL-1, and IL-6: a meta-analysis. Psychosom. Med. 71, 171-186 (2009).

15. Dowlati, Y. et al. A meta-analysis of cytokines in major depression. Biol. Psychiatry 67, 446-457 (2010).
16. Haapakoski, R., Mathieu, J., Ebmeier, K. P., Alenius, H. \& Kivimaki, M. Cumulative meta-analysis of interleukins 6 and 1 beta, tumour necrosis factor alpha and Creactive protein in patients with major depressive disorder. Brain Behav. Immun. 49, 206-215 (2015).

17. Hodes, G. E. et al. Individual differences in the peripheral immune system promote resilience versus susceptibility to social stress. Proc. Natl Acad. Sci. USA 111, 16136-16141 (2014).

18. Menard, C. et al. Social stress induces neurovascular pathology promoting depression. Nat. Neurosci. 20, 1752-1760 (2017).

19. Banks, W. A., Kastin, A. J. \& Gutierrez, E. G. Penetration of interleukin-6 across the murine blood-brain barrier. Neurosci. Lett. 179, 53-56 (1994).

20. Blumberg, J. B., Ding, E. L., Dixon, R., Pasinetti, G. M. \& Villarreal, F. The science of cocoa flavanols: bioavailability, emerging evidence, and proposed mechanisms. Adv. Nutr. 179, 53-56 (2014).

21. Dubner, L., Wang, J., Ho, L., Ward, L. \& Pasinetti, G. M. Recommendations for development of new standardized forms of cocoa breeds and cocoa extract processing for the prevention of Alzheimer's disease: role of cocoa in promotion of cognitive resilience and healthy brain aging. J. Alzheimers Dis. 48, 879-889 (2015).

22. Wang, J. et al. Role of standardized grape polyphenol preparation as a novel treatment to improve synaptic plasticity through attenuation of features of metabolic syndrome in a mouse model. Mol. Nutr. Food Res. 57, 2091-2102 (2013).

23. Wang, J. et al. Grape-derived polyphenolics prevent Abeta oligomerization and attenuate cognitive deterioration in a mouse model of Alzheimer's disease. J. Neurosci. 28, 6388-6392 (2008).

24. Wang, J. et al. Grape derived polyphenols attenuate tau neuropathology in a mouse model of Alzheimer's disease. J. Alzheimers Dis. 22, 653-661 (2010).

25. Wang, J., et al. Targeting multiple pathogenic mechanisms with polyphenols for the treatment of Alzheimer's disease-experimental approach and therapeutic implications. Front. Aging Neurosci. https:/doi.org/10.3389/ fnagi.2014.00042 (2014).

26. Wang, J. et al. Brain-targeted proanthocyanidin metabolites for Alzheimer's disease treatment. J. Neurol. Sci. 32, 5144-5150 (2012).

27. Liu, P. et al. Grape seed polyphenolic extract specifically decreases $A \beta^{*} 56$ in the brains of Tg2576 mice. J. Alzheimer's Dis. 26, 657-666 (2011).

28. Wang, J. et al. Epigenetic modulation of inflammation and synaptic plasticity promotes resilience against stress in mice. Nat. Commun. 9, 477 (2018).

29. Scholey, A. \& Owen, L. Effects of chocolate on cognitive function and mood: a systematic review. Nutr. Rev. 71, 665-681 (2013).

30. Sathyapalan, T., Beckett, S., Rigby, A. S., Mellor, D. D. \& Atkin, S. L. High cocoa polyphenol rich chocolate may reduce the burden of the symptoms in chronic fatigue syndrome. Nutr. J. 9, 55 (2010).

31. Mao, T. K. et al. The effect of cocoa procyanidins on the transcription and secretion of interleukin 1 beta in peripheral blood mononuclear cells. Life Sci. 66, 1377-1386 (2000)

32. Mao, T. K., van de, W. J., Keen, C. L., Schmitz, H. H. \& Gershwin, M. E. Modulation of TNF-alpha secretion in peripheral blood mononuclear cells by cocoa flavanols and procyanidins. Dev. Immunol. 9, 135-141 (2002).

33. Selmi, C., Mao, T. K., Keen, C. L., Schmitz, H. H. \& Eric, G. M. The antiinflammatory properties of cocoa flavanols. J. Cardiovasc. Pharmacol. 47, S163-S171 (2006).

34. Krishnan, V. et al. Molecular adaptations underlying susceptibility and resistance to social defeat in brain reward regions. Cell 131, 391-404 (2007).

35. Golden, S. A., Covington, H. E. III, Berton, O. \& Russo, S. J. A standardized protocol for repeated social defeat stress in mice. Nat. Protoc. 6, 1183-1191 (2011).

36. Hribar, U. \& Ulrih, N. P. The metabolism of anthocyanins. Curr. Drug Metab. 15, 3-13 (2013).

37. Lambert, J. D., Sang, S., Lu, A. Y. \& Yang, C. S. Metabolism of dietary polyphenols and possible interactions with drugs. Curr. Drug Metab. 8, 499-507 (2007).

38. Wang, D. et al. Role of intestinal microbiota in the generation of polyphenolderived phenolic acid mediated attenuation of Alzheimer's disease betaamyloid oligomerization. Mol. Nutr. Food Res. 59, 1025-1040 (2015).

39. Renault, P. F. et al. Psychiatric complications of long-term interferon alfa therapy. Arch. Intern. Med. 147, 1577-1580 (1987).

40. Butler, M. S. Natural products to drugs: natural product-derived compounds in clinical trials. Nat. Prod. Rep. 25, 475-516 (2008).

41. Newman, D. J. \& Cragg, G. M. Natural products as sources of new drugs over the last 25 years. J. Nat. Prod. 70, 461-477 (2007). 
42. Scholey, A. B. et al. Consumption of cocoa flavanols results in acute improvements in mood and cognitive performance during sustained mental effort. J. Psychopharmacol. 24, 1505-1514 (2010).

43. Muller, J., Dettmer, D. \& Macht, M. The Attitudes to Chocolate Questionnaire: psychometric properties and relationship to dimensions of eating. Appetite $\mathbf{5 0}$, 499-505 (2008).
44. van Praag, H. et al. Plant-derived flavanol (-)epicatechin enhances angiogenesis and retention of spatial memory in mice. J. Neurosci. 27, 5869-5878 (2007).

45. Alanon, M. E., Castle, S. M., Siswanto, P. J., Cifuentes-Gomez, T. \& Spencer, J. P. Assessment of flavanol stereoisomers and caffeine and theobromine content in commercial chocolates. Food Chem. 208, 177-184 (2016). 